\title{
CUERPOS EXTRAÑOS INTRAVESICALES. REVISIÓN DE LA LITERATURA
}

\author{
D. PASCUAL REGUEIRO, A. GARCÍA DE JALÓN MARTÍNEZ, E. MALLÉN MATEO, \\ C. SANCHO SERRANO, A. GONZALVO IBARRA, L.A. RIOJA SANZ
}

Servicio de Urología. Hospital Universitario Miguel Servet. Zaragoza.

Actas Urol Esp. 27 (4): 265-273, 2003

\section{RESUMEN}

"CUERPOS EXTRAÑOS INTRAVESICALES. REVISIÓN DE LA LITERATURA"

Queremos hacer una revisión de la literatura sobre los cuerpos extraños intravesicales.

Esta situación clínica no es una urgencia habitual, sin embargo hay gran cantidad de referencias en los artículos urológicos.

En esta revisión queremos compilar los síntomas, los diferentes orígenes de los cuerpos extraños, los métodos de diagnóstico y tratamiento de esta patología.

También queremos añadir a esta revisión nuestra experiencia en un caso reciente.

PALABRAS CLAVE: Cuerpos extraños intravesicales.

\section{ABSTRACT \\ "BLADDER FOREIGN BODIES. BIBLIOGRAPHIC REVIEW"}

We want to make a literature review about the bladder foreign bodies.

This clinical situation is not an usual emergency, but there are a lot of references in urological articles about it.

In this article review we want to make a compilation about the symtoms, the different origins of the foreign bodies, and ways of diagnosis and treatment of this pathology.

We also want to add our personal experience in a recent case.

KEYWORDS: Bladder foreign bodies.

$\mathrm{L}$ a presencia de cuerpos extraños intravesicales no es una urgencia frecuente en el ámbito urológico. No obstante las referencias en la literatura son muy abundantes y de lo más variado en cuanto al origen de dichos cuerpos extraños, motivos de su aparición en vejiga, naturaleza de dichos objetos, forma clínica de presentación y medios aplicados para la extracción de los mismos y resolución del caso de la forma menos cruenta para el paciente.

Con motivo de la presentación recientemente en nuestro Servicio de un nuevo caso de cuerpo extraño intravesical, llevamos a cabo una revisión de la literatura sobre este tema.
No ocultar nuestra sorpresa ante la gran cantidad de referencias bibliográficas sobre esta patología, y su diferente y variada naturaleza, origen y tratamiento.

Sin otro ánimo que el de realizar una mínima síntesis que pueda servir de referencia futura, realizamos en la discusión una visión general de los principales puntos de interés de esta peculiar patología urológica.

\section{DISCUSIÓN}

Como hemos comentado al principio, la literatura es amplia en cuanto a referencias de hallazgos, ya sean casuales, o por presentar clínica, de cuerpos extraños en el interior de la vejiga. 
Las implicaciones de estos cuerpos extraños vesicales van más allá del mero caso clínico de la práctica urológica, ya que pueden estar implicados condicionantes psicológicos, personas con alteraciones psiquiátricas que llevan a cabo prácticas autolesivas, condicionantes sociales, maniobras evasivas del personal del ámbito carcelario, o lesiones genitourinarias en menores de edad, condicionantes jurídicos, lesiones por armas de fuego, etc.

Tampoco hay que olvidar que, debido a las implicaciones ético-morales que lleva implícito esta patología, el diagnóstico, cuando no casual, puede ser complicado, por el no reconocimiento de la persona del acto, o la imposibilidad para sospecharlo. Por este motivo en muchas ocasiones el diagnóstico se demora incluso durante años presentando el paciente síntomas prolongados que no desaparecen a pesar de maniobras terapéuticas.

También, y como apunte inicial, me gustaría hacer hincapié en el derecho a la privacidad del paciente y el secreto profesional inherente a nuestra profesión, que dado el carácter erótico-sexual que acompaña en muchas ocasiones a esta patología, y la angustia del paciente ante una situación tan comprometida, es si cabe, más necesario.

\section{Paciente}

Cuando en un ámbito médico se comenta el caso clínico de extracción de un cuerpo extraño vesical, uno de los primeros motivos que nos vienen a la mente para la introducción de ese cuerpo extraño es el sexual.

Sin embargo son muchos los pacientes que han sido diagnosticados y tratados por este motivo y cuyo origen es bien distinto (ver Tabla II).

Si hacemos referencia a los objetos que voluntariamente son introducidos en la vejiga por el propio paciente, el fin erótico-sexual es uno de los motivos más frecuentes reflejados en la literatura. La brevedad de la uretra femenina, la utilización de objetos de pequeño calibre, como es obvio, determina que el objeto escape en un momento dado del control de la persona, ayudado por la contracción de los músculos perineales introduciéndose en vejiga, y siendo por lo tanto imposible de recuperar. En el caso que se nos presentó recientemente, se trataba de una chica de 21 años, para- pléjica por una ataxia de Friedrich, que se había introducido con este fin un palo de plástico perteneciente a un caramelo de tipo "chupa-chups" (Figs. 1 y 2).

En el caso de varones el mecanismo es el mismo habiendo más riesgo de daños inmediatos o secuelas posteriores a nivel uretral.

Otro grupo de pacientes serían aquellos con alteraciones psiquiátricas, como son el retraso mental y la esquizofrenia, que bien con los fines erótico-sexuales, o bien con fines autolesivos llevan a cabo la introducción, a veces compulsiva y reiterativa de objetos en la vejiga, que pueden pasar desapercibidos durante años.

Un subgrupo de estos pacientes serían aquellos que bajo el efecto de drogas o alcohol y en el contexto sexual, llevan a cabo la introducción, por ellos mismos o por su pareja, de objetos a través del meato uretral, que en ocasiones no alcanza la vejiga pero que puede producir importantes daños en la uretra.

La literatura describe también los cuerpos extraños intravesicales en niños que, con fines autoexploratorios, y al igual que se introducen objetos en el conducto auditivo o en las fosas nasales, lo hacen a través de la uretra no pudiendo recuperarlo.

Por otro lado tenemos a un grupo de pacientes, que siendo sometidos a maniobras urológicas, endoscópicas o no, presentan restos de los materiales en su interior, por olvido de los mismos, rotura inadvertida, migración desde otros lugares etc.

Podríamos considerar a un subgrupo de estos últimos, a aquellos pacientes que tras alguna instrumentación médica no urológica han sufrido una migración de materiales que han acabado en la vejiga.

Por último nombrar los pacientes que presentan un cuerpo extraño intravesical de causa accidental, sin que ningún fin propio o iatrogénico haya mediado.

\section{Origen del cuerpo extraño}

Muchos y muy variados son los cuerpos extraños, recogidos por orden en la Tabla I, que han sido identificados en el interior de la vejiga, bien directamente, o en forma de una calcificación o litiasis conteniendo el objeto en su interior. 

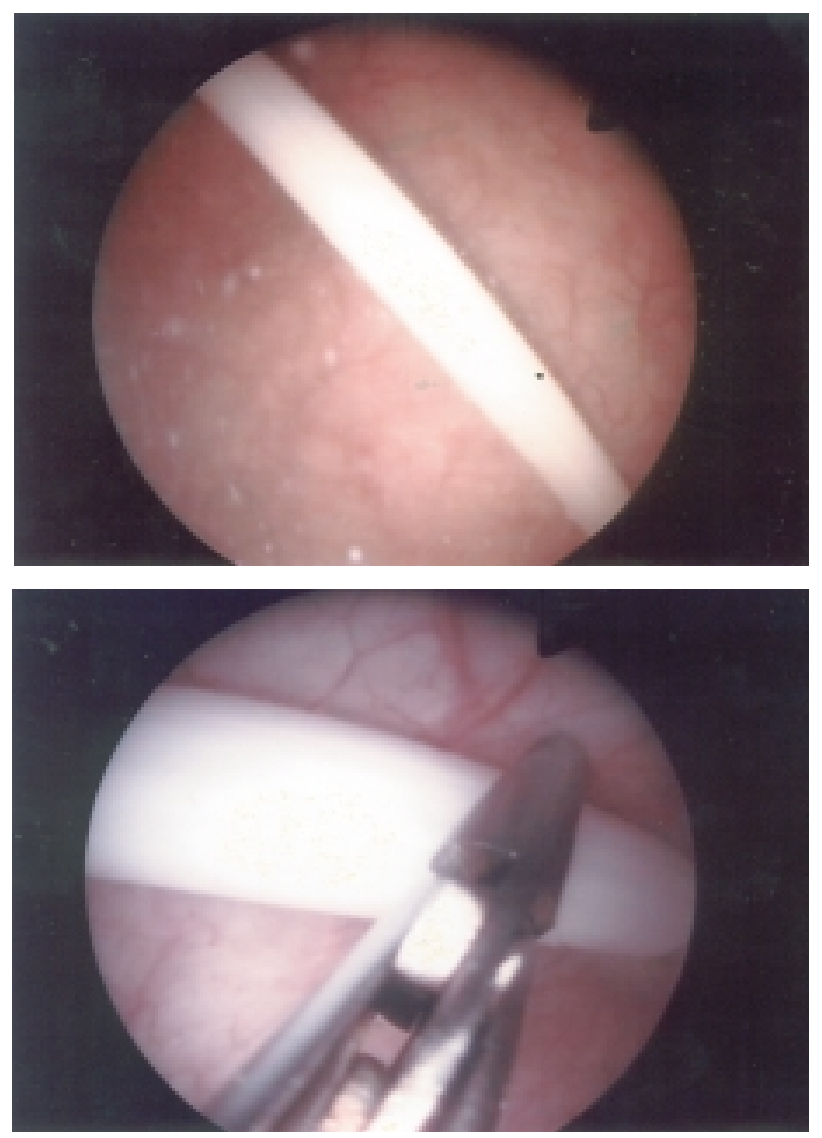

FIGURA 1. En la imagen endoscópica superior se identifica un objeto alargado de color blanco y superficie estriada correspondiente al palo de un caramelo de tipo "chupachups". En la imagen endoscópica inferior, observamos un intento de hacer presa sobre el objeto con unas pinzas de cuerpos extraños.

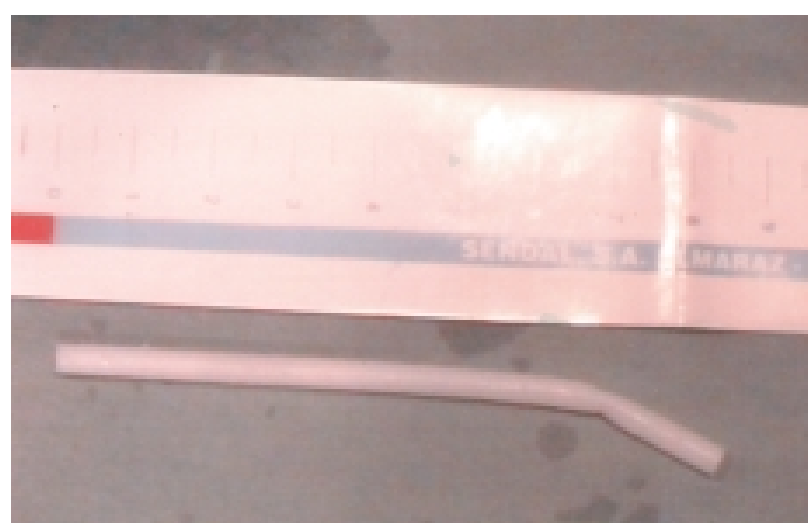

FIGURA 2. Comprobación de la extracción completa del objeto y medición del mismo. Aproximadamente $9 \mathrm{~cm}$.

- El primer grupo está constituido por los objetos introducidos directamente en el interior de la vejiga.
TABLA I

CLASIFICACIÓN DE LOS CUERPOS EXTRAÑOS INTRAVESICALES SEGÚN SU ORIGEN

\section{- INTRODUCIDOS DIRECTAMENTE EN LA VEJIGA}

- Por la propia persona

Entre otros:

Horquillas de pelo, imperdibles, lapiceros, minas, monedas, cargas de bolígrafo, condones,

cables de luz, de teléfono, hilos de cobre, fragmentos de plástico, tornillos, clavos,

huesos de alimentos, sedal de pesca, munición, barras de plástico y de metal, pelos,

cadenas de cuentas, palos de caramelo ("chupachups"), etc.

\section{- Accidentalmente}

Balas tras disparo abdominal

\section{- latrógenos}

\section{Dobles J}

Fragmentos de sonda vesical

Fragmentos de catéteres

Fragmentos de material endoscópico

Suturas y grapas quirúrgicas

Termómetros

\section{- MIGRADOS DESDE OTROS LUGARES:}

- De origen urológico

Partes de prótesis de incontinencia

Partes de prótesis de pene

Grapas quirúrgicas

Puntos de suspensión vesical de técnicas de tratamiento de la incontinencia

\section{- De origen ginecológico}

DIUs

Clips de cirugia de ligadura de trompas

Pesarios de silicona

\section{- De origen digestivo}

- Ingerido y fistulizado

Espina de pescado

Cuchilla de afeitar

- Material quirúrgico

Grapas quirúrgicas

Mallas sintéticas de corrección de

hernias

Prótesis digestivas endoluminales

\section{- De origen vascular}

Prótesis vasculares

Introducidos por la propia persona, con los fines ya descritos, son múltiples y de naturaleza tan dispersa, que sería tedioso hacer una enumeración completa, siendo el único requisito necesario el tamaño del objeto.

En el caso expuesto anteriormente fue utilizado el palo de un caramelo tipo "chupa-chups".

Caso curioso es el de la extracción de una cadena de cuentas utilizada por los soldados noruegos 
para contar los días restantes de servicio ${ }^{27}$, o el de una gruesa litiasis de 360 gramos que albergaba en su interior tres metros de hilo de nylon de pesca.

Introducidos de forma iatrogénica, están descritos restos de material urológico, como fragmentos calcificados de globos de sondas de Foley, fragmentos de catéteres ureterales o dobles $\mathrm{J}$ rotos durante su extracción, o fragmentos de guías metálicas.

Varios artículos hacen referencia al hallazgo de termómetros ${ }^{14}$ en el interior de la vejiga, como complicación accidental en la toma de temperatura vía vaginal.

Introducido directamente en la vejiga, pero por un mecanismo muy distinto, es el hallazgo de proyectiles $^{4,15,19}$. Están descritos casos de agresiones por arma de fuego en el que no es hallado el proyectil apareciendo posteriormente en el interior de la vejiga, existiendo el caso descrito de una hematuria macroscópica cuatro años tras la agresión, que llevó al diagnóstico. Y más llamativo, si cabe, es la expulsión espontánea de proyectiles de pequeño calibre tiempo después de la agresión, sin haberse siquiera sospechado la lesión vesical.

El segundo grupo importante estaría constituido por objetos que han sido encontrados en el interior de la vejiga, después de haber migrado desde otros lugares del ámbito urológico o no.

Así está descrita la erosión e intravesicalización de componentes de prótesis peneanas ${ }^{20}$.

Más llamativo es el hallazgo calcificado intravesical del reservorio de una prótesis de incontinencia AMS $800^{10}$, tras la retirada, trece meses antes por erosión uretral, del componente esfinteriano.

También están descritos los clips quirúrgicos empleados en cirugías de prostatectomía o cistoprostatectomías radicales, o puntos de material irreabsorbible empleados en cirugías de cervicosuspensión para el tratamiento de la incontinencia, que suelen manifestarse en forma de litiasis vesicales $^{22}$ (Tabla I).

Ampliamente descritos están en la literatura los casos clínicos de migración al interior de la vejiga de dispositivos intrauterinos (DIU) ${ }^{7,17}$, que por otro lado son difíciles de diagnosticar si no dan clínica porque su situación anatómica hace que los controles radiológicos no sean sugestivos.
Como curiosidad dentro del ámbito ginecológico, hacer referencia al hallazgo de una fístula vesicovaginal en una anciana, que había sido provocada por el abandono durante años de un pesario vaginal de tres pulgadas de diámetro (unos $7,5 \mathrm{~cm}$ ) y que requirió una amplia rafia para solucionarla ${ }^{11}$.

Más sorprendentes, si cabe, son los casos de migración de material de sutura o protésico desde aparato digestivo, pared abdominal o vascular. Así podemos encontrar decúbitos e intravesicalizaciones producidas por mallas empleadas en la corrección de hernias ${ }^{23}$, gasas olvidadas en el campo quirúrgico, material protésico vascular o digestivo endoluminal, siendo además curioso el hecho de que en muchas ocasiones, dicha migración pasa desapercibida.

Uno de los casos de mayor impacto es la expulsión de una litiasis a través de una fístula vesicovaginal, conteniendo en su interior una cuchilla, que previamente había sido ingerida por la paciente y que había fistulizado, sin clínica, desde el intestino a la vejiga ${ }^{26}$.

\section{Clínica y medios diagnósticos}

Tanto por el hecho de que la presencia de parte de los cuerpos extraños intravesicales son ignorados por el paciente ante la falta de clínica y su origen insospechado (restos de materiales en la vía urinaria, migraciones desde otros puntos etc), como por el hecho del trasfondo personal (fines sexuales que llevan a rehusar atención médica por vergüenza mientras el objeto no da clínica, introducción por personas con alteraciones psiquiátricas o bajo el efecto de sustancias tóxicas), la clínica más frecuentemente expresada es el hallazgo casual del citado cuerpo extraño al realizar alguna prueba de imagen, como una radiografía simple o una ecografía abdominal ${ }^{9}$ (Tablas II, III y IV).

Esto puede ser llevado al extremo, existiendo múltiples casos descritos de extracción del cuerpo extraño varios años después de su introducción, siendo el caso más llamativo, el de la extracción de varios fragmentos de hilo de cobre, en forma de litiasis, 27 años después ${ }^{16}$.

Como es lógico, existe un grupo de pacientes, los cuales tras la introducción voluntaria accidental del cuerpo extraño, acuden alarmados a los 


\section{TABLA II}

CARACTERÍSTICAS DE LOS PACIENTES CON CUERPOS EXTRAÑOS EN VEJIGA
- Niños
- Adultos con alteraciones psiquiátricas
- Personas en ambientes carcelarios
- Personas bajo el efecto de drogas y/o alcohol
- Adultos con fines erótico-sexuales
- Accidentes
- Personas sometidas a instrumentación, tratamientos quirúrgicos, colocación de prótesis

\section{TABLA III}

- MANIFESTACIONES CLÍNICAS DEL CUERPO EXTRAÑo INTRAVESICAL

- Hallazgo casual (Rx, ECO)

- Síntomas urinarios:

- Infecciones urinarias de repetición resistentes a la antibioterapia

- Litiasis vesical, incluso de gran tamaño

- Polaquiuria

- Dolor miccional

- Escozor miccional

- Hematuria

- S. febril asociado a sintomatología urinaria

- MENOS FRECUENTES

- Sospecha de imagen tumoral en pruebas diagnósticas de imagen

- Alteración de la función renal

- Fístula vesicointestinal

- Fístula vesicovaginal

- Expulsión espontánea

\section{TABLA IV}

- PRUEBAS DIAGNóSTICAS MÁS FRECUENTEMENTE
REALIZADAS
- Rx abdomen
- ECO abdominal
- Uretrocistoscopia

- Menos fRecuente

- TAC

- RNM

- Expulsión espontánea

- Hallazgo tras laparotomía

- Hallazgo en autopsia servicios de urgencias relatando lo sucedido, llevándose a cabo, como fue en nuestro caso, la extracción del mismo.

Tras este primer grupo, tenemos en importancia, por otro lado lógica, las manifestaciones clínicas en forma de síntomas urinarios.

Quizá, una de las manifestaciones urológicas más frecuente, es el hallazgo casual o motivado por la clínica, de una o varias litiasis vesicales, siendo la posterior fragmentación de las mismas, lo que revela la presencia del cuerpo extraño en su interior.

Litiasis que en ocasiones pueden alcanzar tamaños desmesurados, como el caso relatado anteriormente de un hilo de sedal de tres metros de longitud, que se convirtió en una litiasis de 10,5 x 7,5 x 7,5 cm, y un peso aproximado de $360 \mathrm{gr}$.

Las manifestaciones clínicas no han de presentarse inmediatamente, pudiendo aparecer meses después en forma de molestias en hipogastrio, polaquiuria, dolor y/o escozor al orinar, hematuria o bien síndrome febril asociado a sintomatología de tracto urinario inferior.

Así, la aparición de una hematuria macroscópica, puso de manifiesto la presencia de un proyectil intravesical, cuatro años después de la agresión por arma de fuego, y asintomática hasta entonces.

Muy frecuentemente, la presencia de cuadros infectivos urinarios reiterativos, que no acaban de responder a la antibioterapia, y provocados por cepas multirresistentes de gérmenes como el Proteus mirabillis o Pseudomona aeruginosa, pueden estar poniéndonos en la pista de la presencia de un cuerpo extraño intravesical, que en muchas ocasiones se manifestará en forma de litiasis.

Mucho menos frecuente y debido a cuerpos extraños de gran volumen y calcificados, es el establecimiento de una insuficiencia renal de causa obstructiva, por compromiso del drenaje urinario a vejiga, lo que en algún caso descrito, ha determinado una insuficiencia renal crónica por hidronefrosis.

Debido a las características morfológicas de los objetos introducidos en vejiga, o migrados desde otras zonas, es poco frecuente su posterior expulsión espontánea, si bien podríamos encontrar un sesgo de información, dado que aquellos pacientes que hubieran introducido pequeños cuerpos 
extraños en su vejiga, y que posteriormente los hubieran expulsado con la micción, al igual que se expulsan cálculos, a veces de gran tamaño, lógicamente no acudirían para recibir atención médica, y no se tendría constancia de ellos.

No obstante, están recogidos en la literatura casos de expulsión espontánea de cuerpos extraños, de los cuales incluso el propio paciente ignoraba su existencia.

Casos más llamativos, y que para nada pueden hacer pensar en un objeto intravesical son por ejemplo la simulación de lesiones tumorales, que obligan incluso a la laparotomía exploradora y excisión del tejido sospechoso hallando en su interior dicho objeto. Como ejemplo de esta situación está descrito la exéresis de una masa uracal con el diagnóstico preoperatorio de sospecha de absceso uracal, y con el diagnóstico final de una espina de pescado que había fistulizado desde intestino quedando enclavada en el uraco, originando un xantogranuloma perivesical ${ }^{2}$.

La presencia de neumaturia, fecaluria o la pérdida de orina a través de vagina, son signos inequívocos de la existencia de una fístula entero-vesical y vesicovaginal respectivamente, patologías éstas, que en ocasiones llevan asociadas la migración de cuerpos extraños desde la vía digestiva tras su ingestión, o de la vía urinaria a la genital ${ }^{5,6,8}$.

Dichas situaciones en ocasiones pasan desapercibidas, pero en otras pueden originar cuadros sépticos muy graves que pueden comprometer seriamente la vida del enfermo, y obligarnos a llevar a cabo intervenciones de urgencia con gran morbi-mortalidad asociada.

En lo que hace referencia al diagnóstico, es fundamental la correcta anamnesis para poder identificar los síntomas cuando los halla y cuando sea posible llevarla a cabo en dependencia de las características psíquicas del paciente.

Generalmente, cuando el paciente acude por voluntad propia tras la introducción del cuerpo extraño, admite dicha introducción, pero en ocasiones puede referir sintomatología inespecífica o centrada sobre el aparato urinario buscando un diagnóstico "involuntario". Por este motivo es importante un ambiente de privacidad, si bien no siempre es posible, sobre todo si se trata de pacientes jóvenes que vienen acompañados por sus familiares, como ocurrió en nuestro caso.
También es fundamental una vez admitido el acto, precisar bien sobre las características y número de objetos introducidos, para poder planificar de forma adecuada el tratamiento.

En el caso de pacientes con alteraciones psiquiátricas la anamnesis del paciente puede ser complicada debiendo recurrir si es posible a la de las personas responsables.

Lógicamente en el caso de cuerpos extraños no sospechados la anamnesis nos va a dar poca o ninguna información que nos pueda hacer sospechar.

Tras la anamnesis, y partiendo de una sospecha o confirmación de un cuerpo extraño introducido voluntariamente, es importante una exploración genitourinaria para determinar la presencia de lesiones en el meato uretral o lesiones asociadas a nivel genital.

En nuestro caso, la simple realización de un tacto vaginal permitió la identificación de un objeto duro y alargado en profundidad, y en situación transversa.

Las pruebas principales para el diagnóstico de objetos intravesicales, son la radiografía simple de abdomen con proyecciones A-P y oblicuas y la ecografia (Tabla IV), que nos van a poner de manifiesto la existencia de un cuerpo extraño en el interior de la vejiga.

En el caso de la radiografía simple, será preciso el diagnóstico diferencial con artefactos, dispositivos intrauterinos, coprolitos etc., que ofrecen menos dudas en el estudio ecográfico.

Dada las diferentes formas de presentación, y de lo insospechado en ocasiones de esta patología, están descritos estudios de imagen utilizando la tomografía axial computadorizada o la resonancia nuclear magnética, por otro lado infrecuentes.

No obstante, la prueba que demuestra sin ningún género de dudas la presencia de un cuerpo extraño intravesical, es la cistoscopia. Sin embargo, y como se puede apreciar a lo largo de todo el relato, lo que se va a identificar en muchas de las ocasiones va a ser una o varias litiasis, que albergan en su interior el cuerpo extraño.

Tampoco debemos olvidar los hallazgos tras laparotomías exploradoras, o descubrimientos casuales "pos-mortem" durante la realización de una autopsia ${ }^{24}$. 


\section{Extracción del cuerpo extraño ${ }^{1}$}

Una vez identificado el cuerpo extraño intravesical, ya sea de forma accidental, o en el contexto de un cuadro clínico, se impone el tratamiento consistente en la extracción de dicho cuerpo extraño, para ello debemos tener en cuenta varios puntos:

- Sexo.

- Edad.

- Características del cuerpo extraño.

- Tamaño del cuerpo extraño.

- Posibilidad de existencia de lesiones asociadas.

- Existencia de un cuadro clínico asociado.

Asímismo, saber que disponemos de distintas posibilidades para su extracción (ver Tabla V).

- Uretrocistoscopia.

- Cistoscopio rígido.

- Cistoscopio flexible.

- Renoscopio.

- Histeroscopio.

- Endoscopia percutánea.

- Mínima incisión + dilatación +introducción de endoscopio.

- Trocar de laparoscopia.

- Laparoscopia ${ }^{18}$.

- Laparotomía + cistotomía.

- Combinada: visión endoscópica transuretral + extracción percutánea.

Con la premisa fundamental de aplicar siempre el tratamiento menos traumático posible, con menor posibilidad de complicaciones y que a la vez sea el más efectivo y rápido para el paciente, deberemos tener en cuenta los siguientes aspectos:

La extracción endoscópica ${ }^{21,25}$ quizás sea el medio más "fisiológico", por la utilización para la extracción de la vía natural. Además, en el caso de tratarse de una paciente del sexo femenino, puede llevare a cabo sin anestesia, siendo en la mayor parte de los casos muy bien tolerado, hecho éste que agiliza tanto el tratamiento como el alta, que puede ser dada de forma inmediata tras la extracción, como ocurrió en nuestro caso, el cual se realizó con anestesia intruretral pasando la paciente directamente de quirófano a su domicilio con una cobertura antibiótica por la instrumentación (Tabla V).

Sin embargo, la endoscopia puede encontrar una serie de inconvenientes que pueden convertirla en una vía no tan apropiada como podía parecer.

\section{TABLA V}

MÉTODOS UTILIZADOS PARA LA EXTRACCIÓN DE LOS CUERPOS EXTRAÑOS VESICALES

- Uretrocistoscopia

- Cistoscopio rígido

- Cistoscopio flexible

- Renoscopio

- Histeroscopio

- Endoscopia percutánea

- Mínima incisión + dilatación + introducción de endoscopio

- Trocar de laparoscopia

- Laparotomía + cistotomía

- Combinada: visión endoscópica transuretral + extracción percutánea

En el caso de tratarse de un paciente varón, la instrumentación uretral no suele ser tan bien tolerada por características anatómicas obvias, lo que puede estar empeorado por la existencia de una impronta prostática acusada y lo que nos obliga, en muchas ocasiones, al menos a una sedación, lo que conlleva un mínimo de observación del paciente tras la misma, y un ingreso al menos de 24 horas.

El tamaño del cuerpo extraño puede ser por sí mismo un impedimento absoluto para la extracción vía endoscópica, ¿como extremos un ovillo de cable?, ¿o una litiasis de $10 \mathrm{~cm}$ de diámetro?.

En otras ocasiones aunque el tamaño no sea impedimento, la forma del objeto puede sugerirnos la posibilidad de un daño uretral o esfinteriano que puede acarrear problemas ulteriores de estenosis o de incontinencia.

En el caso de niños debería en lo posible evitarse la instrumentación de la uretra, por los riesgos antes referidos y por la dificultad en ocasiones de disponer del material endoscópico adecuado.

Dentro de este punto de discusión, estaría la utilización de cistoscopio rígido o cistoscopio flexible. A favor del primero, tendríamos la mejor visión del campo, mejores canales de trabajo y mayor disponibilidad en los servicios de urología. A favor del segundo tendríamos su carácter menos traumático sobre la uretra.

No obstante, está descrito la utilización incluso de histeroscopios ${ }^{13}$, y como es nuestro caso, la utilización del renoscopio, que nos permitió la utilización de unas pinzas de mayor rigidez para 
poder hacer presa sobre el objeto, ya que los primeros intentos con un cistoscopio compacto del 17 Ch primero, y posteriormente con un cistoscopio del $24 \mathrm{Ch}$, no nos permitió la extracción del cuerpo extraño, dado que al presentar una fina estriación en superficie, las pinzas no hacían presa en él.

Para los casos en los que la extracción endoscópica no sea la opción más indicada, tenemos otras posibilidades, como es la extracción vía percutánea $^{12}$, en la que se puede realizar una dilatación progresiva del orificio de una talla vesical, ya sea con los dilatadores de Alken o los de Amplatz, e introducir posteriormente un cistoscopio. O bien la introducción de un trocar de laparoscopia por vía suprapúbica, a través del cual podemos introducir pinzas, litotriptores, etc., con un apoyo visual a través de un cistoscopio por vía uretral, técnicas que obviamente llevan asociado un acto anestésico.

Por supuesto la cirugía abierta con cistotomía, nos queda siempre como recurso final, siendo de primera indicación en cuerpos extraños de gran tamaño, cuando no existe un diagnóstico seguro de qué tipo de lesión tenemos delante, y por supuesto cuando existen lesiones asociadas como pueden ser fístulas digestivo-urinarias, o fístulas genitourinarias que requieren de una reparación.

Unido al tratamiento del cuerpo extraño, habrá que llevar a cabo el de las complicaciones que puede llevar asociado, como las citadas fístulas, cuadro sépticos que requerirán antibioterapia, alteración de la función renal, alteraciones mentales que requerirán apoyo psiquiátrico, etc.

\section{CONCLUSIONES}

- Los cuerpos extraños intravesicales son una patología urológica, que aunque no frecuente en la práctica diaria, no desdeñable en la bibliografía.

- Personas con fines autoeróticos, pacientes con alteraciones mentales permanentes o transitorias con fines autolesivos, niños llevados por la curiosidad autoexploratoria, y personas sometidas a instrumentaciones quirúrgicas, urológicas o no, son los grupos de pacientes más frecuentes.

- El hallazgo casual es uno de los medios más usuales de diagnóstico.
- Los síntomas urinarios, especialmente en forma de litiasis vesicales o infecciones urinarias recurrentes y multirresistentes al tratamiento antibiótico, son los más frecuentemente relatados por los pacientes.

- El tratamiento fundamental consiste en la extracción del cuerpo extraño vesical, utilizando en cada ocasión el medio menos cruento, sencillo y rápido para el paciente, y que esté sujeto al menor número de complicaciones posibles.

- Para ello disponemos de la endoscopia urinaria, la endoscopia percutánea, técnicas combinadas y la cirugía abierta.

- Conjuntamente a ésto, habrá que tener en cuenta el tratamiento de las posibles complicaciones asociadas, fístulas, lesiones uretrales, alteraciones mentales, etc.

\section{REFERENCIAS}

1. VAN OPHOVEN A, DEKERNION JB.: Clinical management of foreign bodies of the genitourinary tract. $J$ Urol 2000 aug; 164 (2): 274-287.

2. KINEBUCHI Y, NAKAZAWA M, FUJIWARA M, YONEYAMA T.: Urachal xanthogranuloma caused by a swallowed fish bone: a case report. Hinyokika Kiyo 2001 nov; 47 (11): 797-800

3. BRIONES MARDONES G, JIMÉNEZ CIDRE M, SÁENZ MEDINA J, POZO MENGUAL B, SANZ MIGUELANEZ JL, CRUZ GUERRA N.: Cuerpos extraños uretrovesicales. Actas Urol Esp 2001 jun; 25 (6): 456-457.

4. HALKIC N, WISARD M, ABDELMOUMENE A, VUILLEUMIER H.: A large bullet in the bladder. Swiss Surg 2001; 7 (3): 139-140.

5. WYCZOLKOWSKI M, KLIMA W, LABZA $H$, KASPRZYK J.: Vesico-ileal fistula caused by a foreign body. Urol Int 2001; 66 (3): 164-165.

6. MUKOUYAMA H, OGAWA Y, KOYAMA Y.: Vesicorectal fistula due to pelvic foreign body: a case report. Hinyokika Kiyo 2001 feb; 47 (2): 109-111.

7. ATASOY C, FITOZ S, AKYAR S.: Bladder stone caused by an ectopic intrauterine contraceptive device: case report. Can Assoc Radiol J 2001 feb; 52 (1): 33-34.

8. TORNERO J, PALOU J, PRADOS M, SALVADOR J, VICENTE J.: Bladder perforation caused by foreign body migration. Int Urol Nephrol 2000; 32 (2): 241-243.

9. BARZILAI M, COHEN I, STEIN A.: Sonographic detection of a foreign body in the urethra and urinary bladder. Urol Int 2000; 64 (3): 178-180. Urol Int 2000; 64 (3): 167-168.

10. BARTOLETTI R, GACCI M, TRAVAGLINI F, SARTI E, SELLI C.: Intravesical migration of AMS 800 artificial urinary sphincter and stone formation in a patient who underwent radical prostatectomy.

11. GRODY MH, NYIRJESY P, CHATWANI A.: Intravesical foreign body and vesicovaginal fistula: a rare complication of a neglected pessary. Int Urogynecol J Pelvic Floor Dysfunct 1999; 10 (6): 407-408. 
12. HUTTON KA, HUDDART SN.: Percutaneous retrieval of an intravesical foreign body using direct transurethral visualization: a technique applicable to small children. BJU Int 1999 feb; 83 (3): 337-338.

13. SZLYK GR, JARRETT TW.: Use of rigid hysteroscope for extraction of foreign bodies embedded in lower urinary tract. $J$ Endourol 1999 feb; 13 (1): 47-48.

14. RIOU PJ, HARRIS A.: Intravesical thermometer: an unusual complication of vaginal temperature measurernent. J Accid Emerg Med 1998 nov; 15 (6): 432.

15. GULANIKAR A, PANDEY P, TERRELL F.: A bullet in the bladder. Br J Urol 1998 aug; 82 (2): 304.

16. DAVIDOV MI.: A case of the multiyear presence of a foreign body in the bladder. Urol Nefrol (Mosk) 1997 jul-aug; (4): 40-41.

17. HERMIDA PÉREZ JA, DEL CORRAL SUÁREZ T, CERDEIRAS MARTÍNEZ G, AGUERO GÓMEZ JL, MACHADO V.: Bladder lithiasis formed from an IUD. A rare case. Arch Esp Urol 1997 sep; 50 (7): 808-809.

18. EJSTRUD P, POULSEN J.: Laparoscopic removal of an electric wire from the bladder. Urol 1997 aug; 80 (2): 338.

19. DIDOMENICO D, GUINAN P, SHARIFI R.: Spontaneous expulsion of an intravesical bullet. $J$ Am Osteopath Assoc 1997 jul; 97 (7): 415-416.

20. SWANA HS, FOSTER HE Jr.: Erosion of malleable penile prosthesis into bladder. J Urol 1997 jun; 157 (6): 2259-2260.

21. WEGNER HE, FRANKE M, SCHICK V.: Endoscopic removal of intravesical pencils using percutaneous nephrolithotomy sheath and forceps. J Urol 1997 may; 157 (5): 1842 .
22. KESBY GJ, KORDA AR.: Migration of a Filshie clip into the urinary bladder seven years after laparoscopic sterilisation. Br J Obstet Gynaecol 1997 mar; 104 (3): 379-378.

23. HUME RH, BOUR J.: Mesh migration following laparoscopic inguinal hernia repair. $J$ Laparoendosc Surg 1996 oct; 6 (5): 333-335.

24. DERNIEV IN, NIGMATULLIN NSH.: Unusual finding of a foreign body during forensic medical expertise on a cadaver. Sud Med Ekspert 1996 jul-sep; 39 (3): 52-53.

25. GAFFNEY CM.: Endoscopic removal of difficult bladder foreign bodies. J S C Med Assoc 1996 jan; 92 (1): 9-10.

26. PAJOR L, SZABO V.: Bladder stone formation on a swallowed knife blade and spontaneous passage through a vesicovaginal fistula. Br J Uro1 1995 nov; 76 (5): 659.

27. HAAKONSEN P, STEINSVIK E.: What nobody thought possible-a "demobilization chain" gone astray. Foreign body in the urinary bladder -a current differential diagnosis. Tidsskr Nor Laegeforen 1995 mar 30; 115 (9): 1055-1056.

Dr. D. Pascual Regueiro

Avda. Compromiso de Caspe, 27-29, 2º A

50002 Zaragoza

(Trabajo recibido el 25 abril de 2002) 